\title{
Article \\ The Effects of Graphene on the Biocompatibility of a 3D-Printed Porous Titanium Alloy
}

\author{
Xu Sun, Shuang Tong, Shude Yang and Shu Guo *
}

check for updates

Citation: Sun, X.; Tong, S.; Yang, S.; Guo, S. The Effects of Graphene on the Biocompatibility of a 3D-Printed Porous Titanium Alloy. Coatings 2021, 11, 1509. https://doi.org/10.3390/ coatings11121509

Academic Editor: Mercedes Velazquez

Received: 17 November 2021 Accepted: 3 December 2021

Published: 8 December 2021

Publisher's Note: MDPI stays neutral with regard to jurisdictional claims in published maps and institutional affiliations.

Copyright: (c) 2021 by the authors. Licensee MDPI, Basel, Switzerland. This article is an open access article distributed under the terms and conditions of the Creative Commons Attribution (CC BY) license (https:// creativecommons.org/licenses/by/ $4.0 /)$.
Department of Plastic Surgery, The First Hospital of China Medical University, No.155, Nanjing North Street, Shenyang 110001, China; 2018140092@stu.cmu.edu.cn (X.S.); tongshuang@cmu.edu.cn (S.T.); sdyang@cmu.edu.cn (S.Y.)

* Correspondence: sguo@cmu.edu.cn

\begin{abstract}
D-printed titanium (Ti) materials have attracted much attention in the field of bone tissue repair. However, the combination strength of traditional alloy materials with bone tissue is lower, and the elastic modulus is higher than that of natural bone tissue, which makes the titanium alloy susceptible to stress shielding phenomena after implantation. Therefore, it is urgent to find better surface modification technology. In this study, the physical and chemical properties, toxicity, and proliferation of adipose stem cells of composite graphene-coated titanium alloy (Gr-Ti) were investigated using 3D-printed titanium alloy as a material model. Physical and chemical property tests confirmed that 3D printing could produce porous titanium alloy materials; the compressive strength and elastic modulus of the titanium alloy scaffolds were $91 \pm 3 \mathrm{MPa}$ and $3.1 \pm 0.4 \mathrm{GPa}$, matching the elastic modulus of normal bone tissue. The surface characterization shows that graphene can be coated on titanium alloy by a micro-arc oxidation process, which significantly improves the surface roughness of titanium alloy. The roughness factor ( $\mathrm{Ra}$ ) of the Ti stent was $4.95 \pm 1.12 \mu \mathrm{m}$, while the Ra of the $\mathrm{Gr}-\mathrm{Ti}$ stent was $6.37 \pm 0.72 \mu \mathrm{m}$. After the adipose stem cells were co-cultured with the scaffold for $4 \mathrm{~h}$ and $24 \mathrm{~h}$, it was found that the Gr-Ti scaffold could better promote the early cell adhesion. CCK-8 tests showed that the number of ADSCs on the G-Ti scaffold was significantly higher than that on the Ti scaffold $(p<0.01)$. The relative growth rate (RGR) of ADSCs in Gr-Ti was grade $0-1$ (non-toxic). In the in vivo experiment of repairing a critical bone defect of a rabbit mandible, the bone volume fraction in the Gr-Ti group increased to $49.42 \pm 3.28 \%$, which was much higher than that in the Ti group $(39.76 \pm 3.62 \%)(p<0.05)$. In conclusion, the porous graphenetitanium alloy promotes the proliferation and adhesion of adipose stem cells with multidirectional differentiation potential, which has great potential for the application of bone tissue engineering in repairing bone defects in the future.
\end{abstract}

Keywords: 3D printing; titanium alloy; graphene; adipose-derived stem cells

\section{Introduction}

Maxillofacial bone defects caused by trauma and tumors are major challenges faced by plastic surgeons, but satisfactory repair materials have not been found yet [1,2]. Titanium alloy, which has been widely used as bone repair material, has obvious demerits. On the one hand, titanium alloy is bioinert and has a low binding strength with bone tissues $[3,4]$. On the other hand, the mechanical strength of titanium alloy is much higher than that of natural bone tissue, making titanium alloy prone to stress shielding after implantation. As a result, the surrounding bone tissue is absorbed, and even leads to the loosening or fracture of the implant, resulting in the instability of the combination of materials and tissues $[5,6]$.

To solve these problems, different titanium alloy surface modification techniques have been proposed to meet the requirements of clinical application, such as implant surface coating, which is loaded with bioactive molecules or drugs to modify the implant surface to promote osseointegration at the implant-bone interface. These methods can effectively 
improve the bioactivity and biocompatibility of implants and promote osseointegration at the implant-bone interface $[7,8]$. On the other hand, in view of the stress shielding phenomenon [9], 3D printing technology opens up a new idea for the personalized manufacturing of hard tissue implant materials. Three-dimensional printing technology can adjust the mechanical properties of the material to better match the tissues of the bone. The porous structure of 3D printing can affect the overall density, strength, and elastic modulus of the metal implant, and adjust the pore size and porosity to match the mechanical properties of the host bone tissue, and thus the stress shielding effect can be effectively reduced or eliminated [10]. In addition, the rough internal and external surface of the porous structure is conducive to the adhesion, proliferation, and differentiation of osteoblasts, and promotes the growth of new bone tissue into the pores, so that the implant and bone tissue form a locked structure, and finally form a whole, which is conducive to the long-term stable fixation of the implant. At the same time, the pores of three-dimensional traffic can enable the free transmission of body fluids, bring nutrients to the growth of new bone, take away metabolic waste, promote the regeneration and reconstruction of tissue, and accelerate the whole repair process [11].

Graphene is an allotrope of carbon with a thickness of only $0.35 \mathrm{~nm}$ and a carboncarbon covalent bond of $0.142 \mathrm{~nm}$. Unique mechanical properties, such as high extensibility, flexibility, and adaptability to regular or irregular surfaces, are suitable for cell adhesion and migration [12]. Graphene has been found to be used in bone tissue engineering applications where graphene coated materials can promote stem cell viability and induce the osteogenic differentiation of stem cells compared to traditional substrates or scaffolds $[13,14]$. Microarc oxidation technology has been widely used in the field of medical materials since it was proposed. With mature technology and a stable effect, graphene bioactive coating can be well compounded on the surface of a titanium alloy substrate [15].

The process of bone tissue repair is accomplished through a series of chain reactions involving pluripotent stem cells, such as proliferation and differentiation, the recognition of the extracellular matrix and signal molecules, the expression of related factors, and targeting. Adipose-derived stem cells (ADSCs), as a popular seed cell in recent years, exist in adipose tissue; ADSCs can maintain self-renewal and have multidirectional differentiation potential. They are more abundant in vivo and easier to obtain and amplify in vitro. [16] A large number of studies with clinical application significance are being carried out in the field of bone tissue repair $[17,18]$.

In a large area of bone defect, stem cells cannot play a role alone, so it is necessary to provide a transitional place for stem cells to induce differentiation in vivo with scaffold materials as the carrier. Titanium alloy has good mechanical properties and corrosion resistance; surface modification technology can retain the original excellent mechanical properties of titanium and its alloys, endowing them with special biological functions, so as to meet the clinical needs [19]. In order to make up for the deficiency of traditional titanium alloy materials in repairing bone tissue defects, the porous titanium alloy prepared by selective laser melting (SLM) technology was used to prepare porous titanium alloy implants with composite graphene coating by micro-arc oxidation technology from both the structure and surface. The effects of composite implants on bone repair and bone integration were evaluated in vivo and in vitro, providing a theoretical basis for the combined application of bone tissue engineering and stem cell therapy and an experimental basis for the development and application of new bone defect repair materials in clinical practice.

\section{Materials and Methods}

\subsection{Material Preparation}

The three-dimensional digital model of porous titanium alloy scaffold material was designed and established by CAD software (Version 21.0, Materialise, Leuven, Belgium). Its external shape was $8 \mathrm{~mm} \times 4 \mathrm{~mm} \times 3 \mathrm{~mm}$ and $4 \mathrm{~mm} \times 4 \mathrm{~mm} \times 3 \mathrm{~mm}$, and the cube was the basic structural unit. The internal porosity was about $70 \%$, and the aperture was about $550 \mu \mathrm{m}$ (Figure 1). The porous titanium alloy scaffolds with the above two sizes 
were prepared by selective laser melting (SLM) technology. The equipment used was 3D Systems (Prox DMP 200, Rock Hill, SC, USA). The Ti6Al4V alloy powder size was no larger than $50 \mu \mathrm{m}$, the laser spot diameter was $70 \mu \mathrm{m}$, the scanning distance was $45 \mu \mathrm{m}$, the laser power was $260 \mathrm{~W}$, the scanning speed was $1200 \mathrm{~mm} / \mathrm{s}$, and the layer thickness was $45 \mu \mathrm{m}$ (Figure 1).
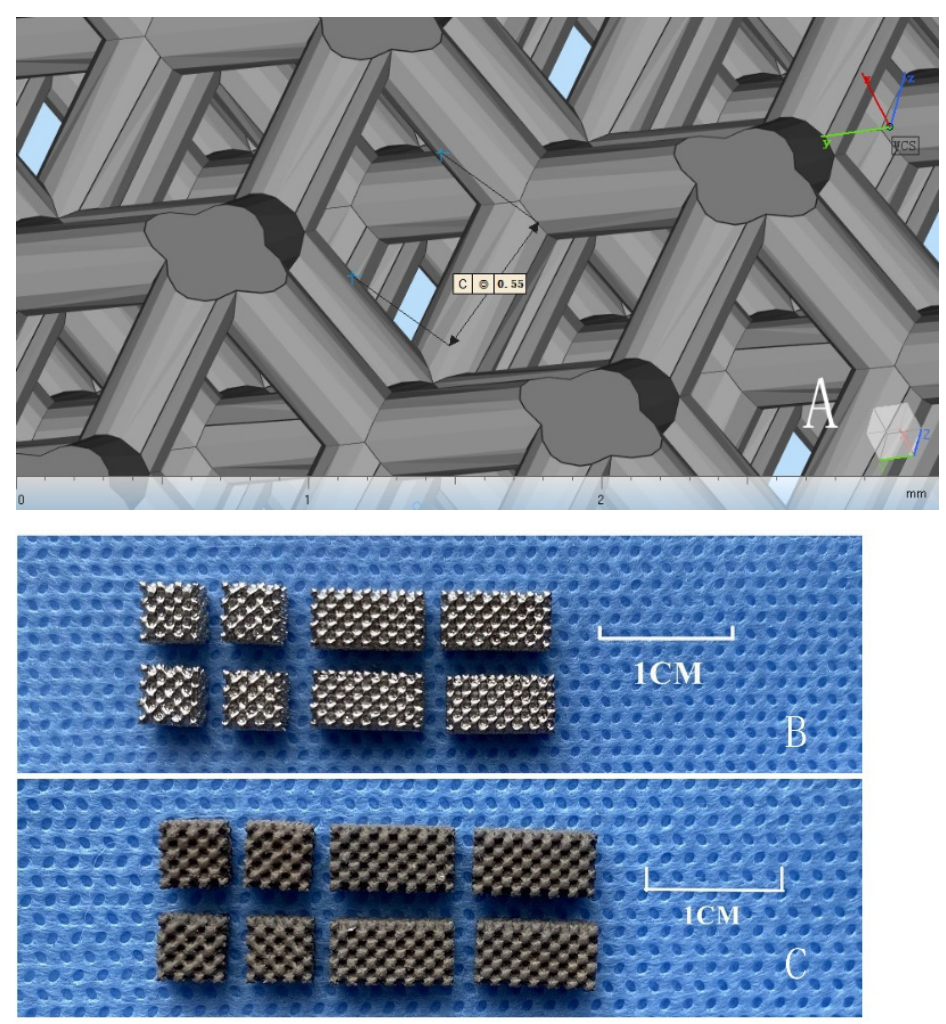

Figure 1. (A) Internal design structure of porous scaffold; (B) porous scaffold without coating; and (C) composite graphene-coated porous scaffold.

Bioactive graphene coatings were prepared on the surface of porous titanium alloy scaffolds by micro-arc oxidation. The pretreated bracket connecting wires were placed in a preprepared electrolyte. The bracket to be processed was used as the anode, a graphite plate was used as the cathode, and the electrolyte was contained in a PTFE tank. The electrolyte used in this experiment was mainly composed of graphene (Nanjing XFNANO Materials Tech Co., Ltd., Nanjing, China), EDTA disodium (Wujiang Aobang Chemical Co., Ltd., Wujiang, China), and deionized water.

\subsection{Determination of the Physical and Chemical Properties of the Scaffolds}

The surface morphologies of the Ti scaffold and Gr-Ti scaffold were compared by scanning electron microscopy (JSM-TM3000, JEOL, Tokyo, Japan). The internal structural parameters such as porosity, pore diameter, and trabecular diameter were examined by scanning electron microscopy. The surface elements of the scaffolds were analyzed by energy-dispersive X-ray spectroscopy (EDS) (JSM-TM3000, Tokyo, Japan) with scanning electron microscopy. A 3D measurement laser confocal microscope (Olympus LEXT OLS4000, Tokyo, Japan) was used to scan and reconstruct the surface topography of the two materials, and their surface roughness was detected and analyzed.

The elastic modulus is $\mathrm{E}=\mathrm{P} /(-\mathrm{dV} / \mathrm{V})$ and the unit is Pa. Compressive strength $\mathrm{P}(\mathrm{MPa})=\mathrm{F}(\mathrm{N}) / \mathrm{S}\left(\mathrm{mm}^{2}\right)$. The material was placed between two pressure plates of the mechanical testing machine. The loading rate of the pressure plate was set as 0.01 $\mathrm{mm} / \mathrm{s}$, and the upper limit of deformation was set as $15 \%$ of the height of the support. 
The elastic modulus was calculated by selecting a relatively flat section of the pressuredeformation curve.

\subsection{Compound Culture and Biocompatibility of ADSCs and Scaffolds In Vitro}

Human adipose tissue was obtained under aseptic conditions and $0.2 \%$ type I collagenase (Sigma Chemical Co., St. Louis, MO, USA) was used for full digestion in a $37^{\circ} \mathrm{C}$ water bath for $45 \mathrm{~min}$. Supernatant and adipose tissue were discarded after centrifugation at $1200 \mathrm{rpm}$ for $5 \mathrm{~min}$ at room temperature. The cells were resuspended by blowing with $15 \%$ FBS and inoculated in a $75 \mathrm{~cm}^{2}$ culture flask at $37^{\circ} \mathrm{C}$ with $5 \% \mathrm{CO}_{2}$ saturated humidity.

CCK-8 assay was used to evaluate the cytotoxicity and proliferation of scaffolds on ADSCs. ADSCs were inoculated with $2 \times 10^{4}$ cells $/ \mathrm{mL}$ on coated and uncoated scaffolds. After $24 \mathrm{~h}$, cells were cultured in the complete medium with 10\% (V/V) CCK-8 reagent (US Everbright Inc., Silicon Valley, CA, USA) for $2 \mathrm{~h}$, and then the spectrophotometry was measured at $450 \mathrm{~nm}$ with a microplate reader. After 1, 4, 7, and 10 days of culture, 6 samples were taken from each time point group, washed with PBS 3 times, and $1 \mathrm{~mL}$ of $0.1 \%$ TritonX-100 was added to each well at $4{ }^{\circ} \mathrm{C}$ overnight, and blown for $1 \mathrm{~min}$. The OD value of the solution in the well was read by the microplate reader at the wavelength of $520 \mathrm{~nm}$.

The morphology of ADSCs in different samples was observed by scanning electron microscopy. ADSCs were inoculated into alloys on 96-well plates with 5000 cells per well. After incubation for 4 and $24 \mathrm{~h}$, the medium was removed. The samples were washed with PBS 3 times and dehydrated in ethanol at 30\%,50\%,75\%,95\%, and 100\% concentrations for $10 \mathrm{~min}$. The samples were fixed with $2.5 \%$ glutaraldehyde at $4{ }^{\circ} \mathrm{C}$ for $4 \mathrm{~h}$ and examined by SEM.

\subsection{In Vivo Experimental Study of Porous Titanium Alloy Scaffolds}

The experimental animals were randomly divided into three groups (6 animals/group): group A was the Gr-Ti group; group B was the Ti group; and group C was used as the blank control group.

After weighing the experimental animals (New Zealand white rabbits, 6 months old, male or female, weight $2-3 \mathrm{~kg})$, they underwent a $10 \%$ chloral hydrate $(3 \mathrm{~mL} / \mathrm{kg})$ abdominal injection of anesthesia. Then, a bilateral area under the jaw and neck skin was disinfected with a shop towel and a transverse incision was made along the edge of the jaw, about $2 \mathrm{~cm}$ long, cutting to the bone surface and completely showing the mandibular lingual nozzles and cheek bone surface. A low-speed turn crack at the edge of the middle mandibular of about $8 \mathrm{~mm} \times 4 \mathrm{~mm}$ created the full-thickness defect of bone. The corresponding scaffolds were implanted, respectively. According to the experimental plan, the animals were sacrificed 4 and 12 weeks after operation, and bilateral mandible specimens were collected.

The postoperative survival status of animals was observed, and inflammatory reactions such as redness, exudation, and ulceration were observed. After sampling, we observed whether the specimen morphology was abnormal, whether the scaffold was combined with surrounding tissues, whether the fibrous granulation tissue was wrapped and adhered, and whether there was prolapse of the scaffold.

Bone ingrowth in the implantable material and bone integration between the material and the tissue were observed and evaluated. Bone ingrowth in the material was quantitatively analyzed by measuring and calculating the bone volume fraction (BVF). BVF was calculated as follows: BVF = volume of newly mineralized bone in the material $\times$ $100 \%$ / pore volume in the material.

SPSS19.0 statistical software was used for the $t$-test, and $p<0.05$ was statistically significant. 


\section{Results}

\subsection{Physical and Chemical Properties of Porous Titanium Alloy Scaffolds}

Figure 1 shows that the porous titanium alloy scaffold has a smooth surface and consistent pores. The surface of the coating specimen is dark and rough without metallic luster.

SEM results are shown in Figure 2. As shown in Figure 2C,D, the surface of the graphene coating shows a uniform microporous structure. The surface is rough and porous relative to the uncoated material (Figure 2A,B). The scanning results showed that the pore parameters of the porous titanium alloy scaffold prepared in this experiment were as follows: porosity $69 \pm 3 \%$, pore diameter $542 \pm 26 \mu \mathrm{m}$, and metal trabecular diameter $244 \pm 21 \mu \mathrm{m}$.
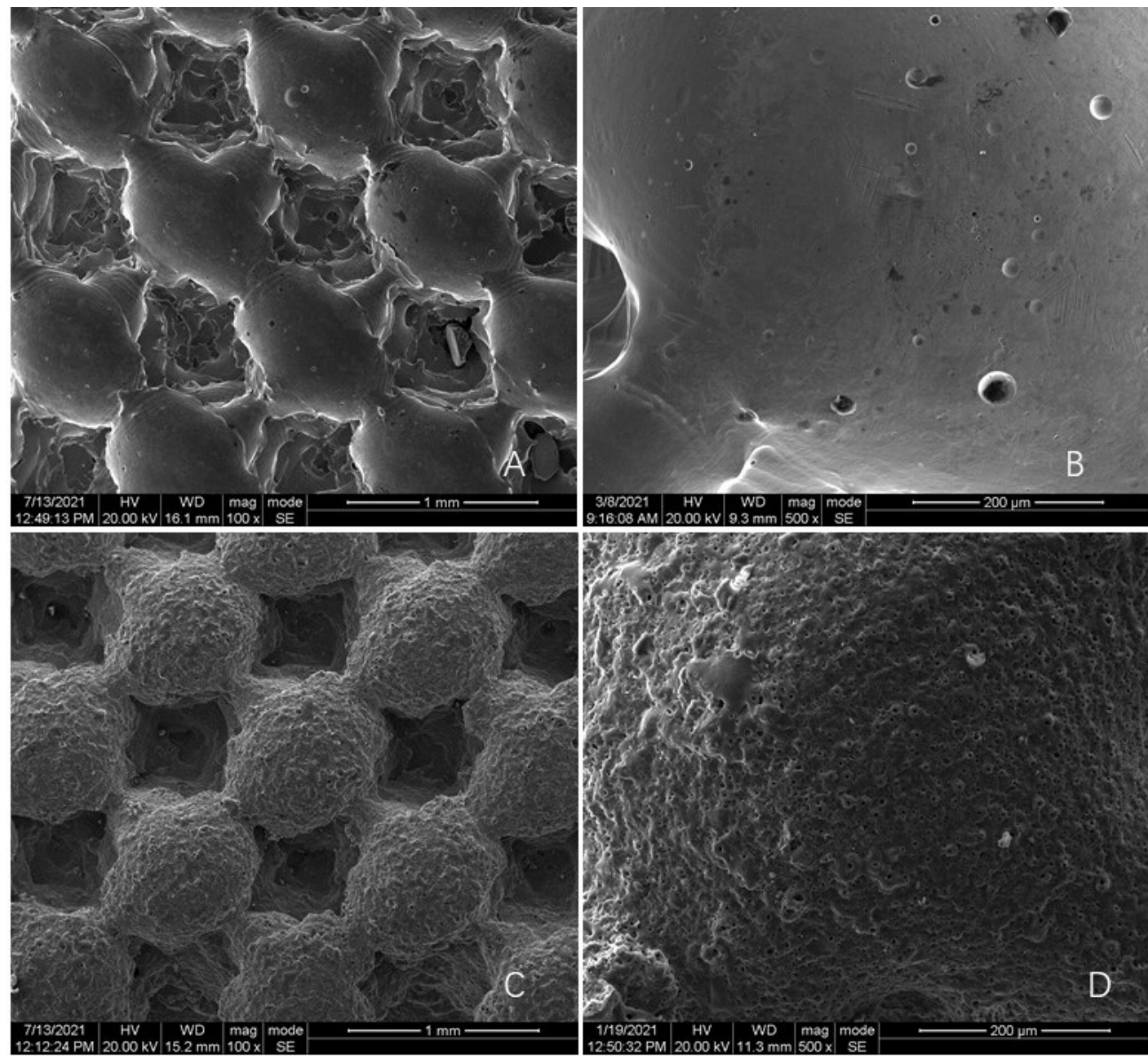

Figure 2. SEM observation of samples: the surface of the graphene coating $(\mathbf{C}, \mathbf{D})$ shows a uniform microporous structure, which is rough and porous relative to the uncoated material $(\mathbf{A}, \mathbf{B})$.

Metallic elements such as titanium and aluminum can be detected by X-ray energy spectrum analysis on the surface of the titanium alloy scaffold (Figure 3A). Elements such as carbon can be detected by micro-arc oxidation (Figure 3B).

The surface roughness is indicated by the average roughness factor (Ra). The Ra of the Ti stent (Figure $4 \mathrm{~A}$ ) was $4.95 \pm 1.12 \mu \mathrm{m}$, while the Ra of the Gr-Ti stent (Figure 4B) was $6.37 \pm 0.72 \mu \mathrm{m}$, with statistically significant differences.

The compressive strength and elastic modulus of the porous titanium alloy scaffolds were $91 \pm 3 \mathrm{MPa}$ and $3.1 \pm 0.4 \mathrm{GPa}$. 

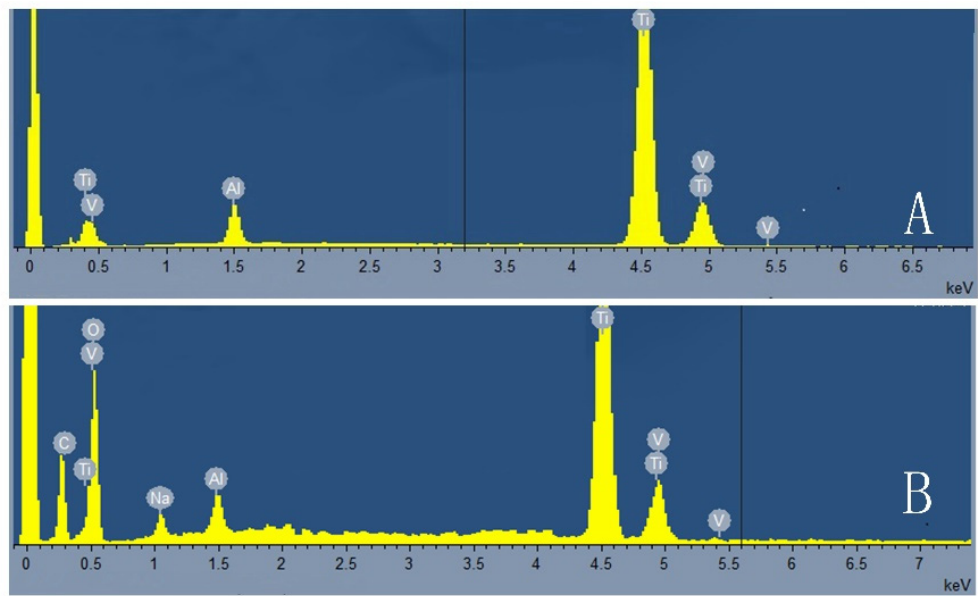

Figure 3. SEM energy dispersive $\mathrm{X}$-ray spectroscopy analysis results: Peak values of $\mathrm{Ti}, \mathrm{Al}, \mathrm{V}$ and other metal elements can be seen on the surface of $\mathrm{Ti}$ (A); carbon can be detected on the surface of Gr-Ti (B).
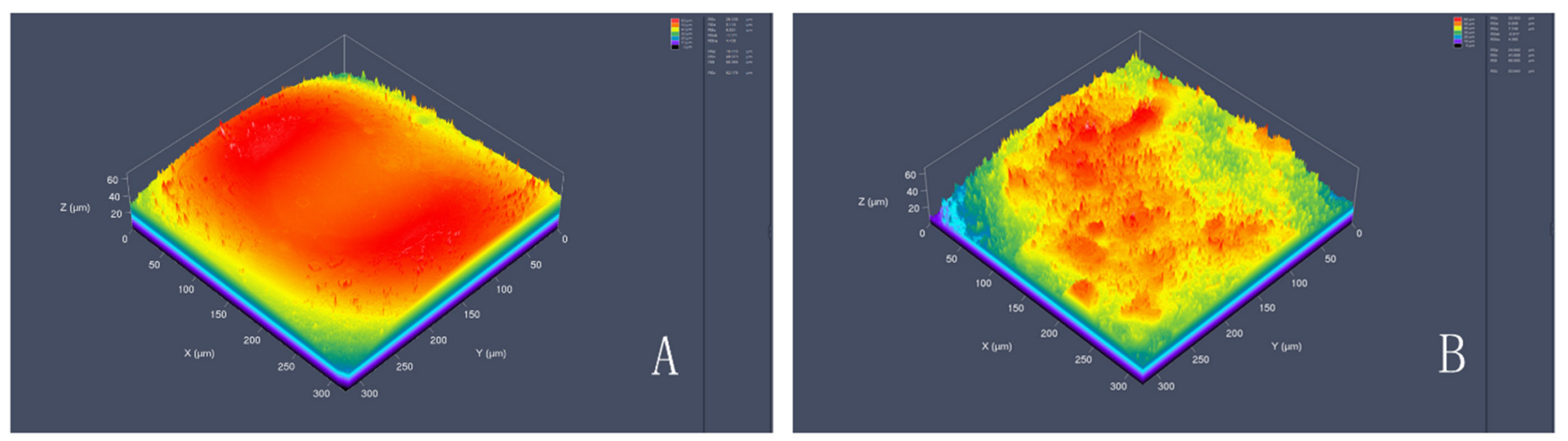

Figure 4. Average roughness: $\mathrm{Ra}$ of $\mathrm{Gr}-\mathrm{Ti}(\mathbf{B})$ was higher than that of $\mathrm{Ti}(\mathbf{A}) ;(p<0.05)$.

\subsection{Adhesion of ADSCs}

As can be seen from Figure 5, at the early stage of culture, at about $4 \mathrm{~h}$, the ADSCs' morphology on the Ti scaffold and Gr-Ti scaffold group was similar without a significant difference, with scattered round or oval ADSC adhesion on the surface of the scaffold, granular protrusions on the surface of ADSCs, and a small amount of granular extracellular matrix around the cells (Figure 5A,C). When cultured for $24 \mathrm{~h}$, the number of ADSCs on the Gr-Ti scaffold was significantly higher than that on the Ti scaffold, and the cell morphology of the two scaffolds was different: The pseudopodia of the cells on the Gr-Ti scaffold extended significantly and grew across the pores of the scaffold, forming an anchor-like structure that firmly bonded with the scaffold and connected with the pseudopodia of other cells. The cells were connected in pieces (Figure 5D).

\subsection{Cell Proliferation and Cytotoxicity}

The proliferation capacity of the ADSCs in each group showed an increasing trend after $24 \mathrm{~h}$ of culture, the number of ADSCs on the G-Ti scaffold was significantly higher than that on the Ti scaffold $(p<0.01)$, and the number of ADSCs on both scaffolds was higher than that on the control group $(p<0.01)$ (Figure 6A). The results showed that graphene coating could promote the proliferation of ADSCs on porous titanium alloy scaffolds. The relative growth rate (RGR) of the ADSCs in all samples is shown in Table 1. The formula is as follows: 


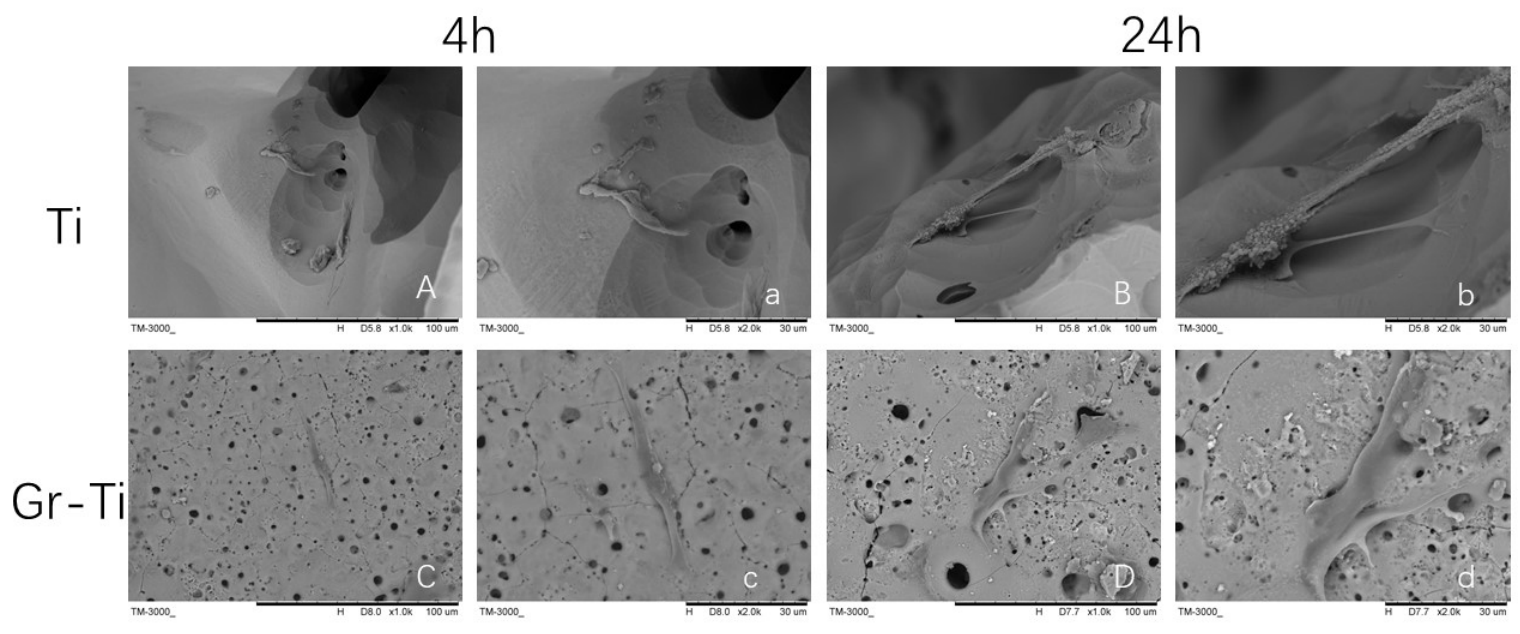

Figure 5. The adhesion of ADSCs on different scaffolds observed by SEM. (A/a,C/c) At 4 h, ADSCs morphology on Ti scaffold and Gr-Ti scaffold group was similar without significant difference. (B/b,D/d) At $24 \mathrm{~h}$, the pseudopodia of the cells on the Gr-Ti scaffold extended significantly and grew across the pores of the scaffold, forming an anchor-like structure.
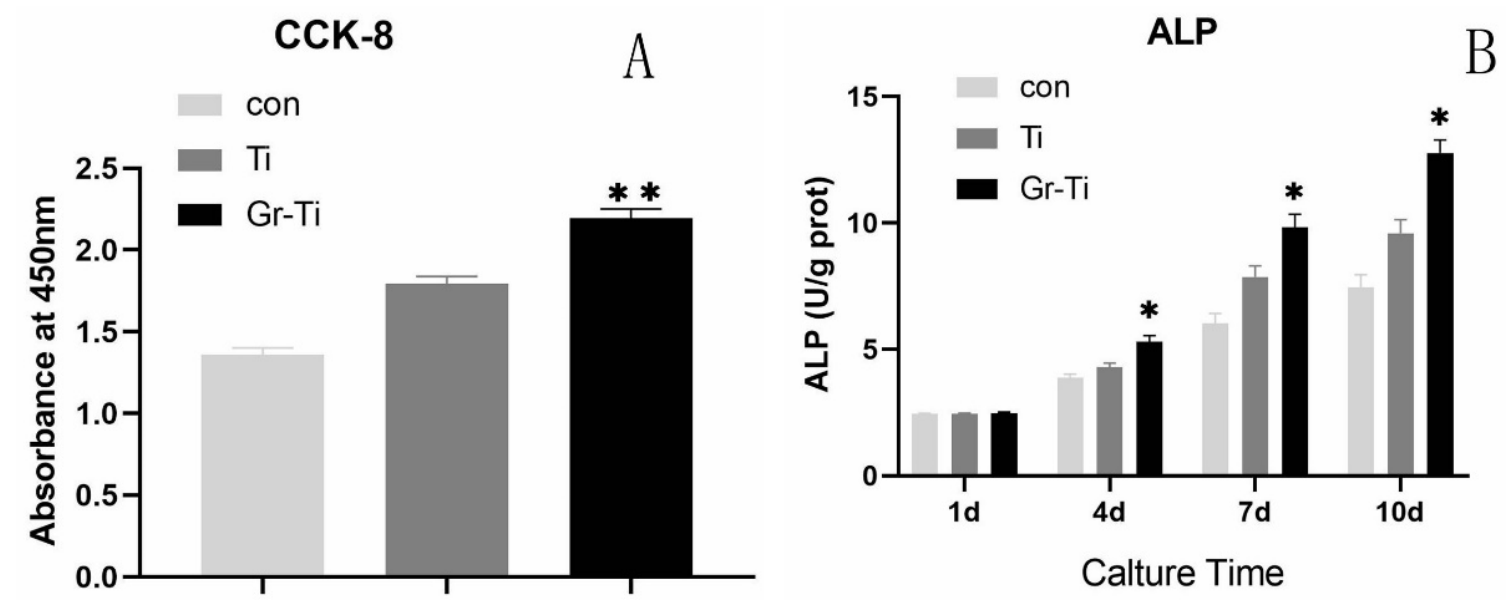

Figure 6. (A) Optical density of ADSCs measured by CCK8 test and (B) ALP test at different detection periods $(n=5$, $\left.{ }^{*} p<0.05,{ }^{* *} p<0.01\right)$.

Table 1. Relative growth rate (RGR) and cytotoxicity level.

\begin{tabular}{ccccccc}
\hline & \multicolumn{2}{c}{$\mathbf{2 4} \mathbf{h}$} & \multicolumn{2}{c}{$\mathbf{4 8} \mathbf{h}$} & \multicolumn{2}{c}{$\mathbf{7 2} \mathbf{h}$} \\
\cline { 2 - 7 } & RGR & Grade & RGR & Grade & RGR & Grade \\
\hline $\mathrm{Ti}$ & $97.63 \pm 1.83$ & 1 & $110.54 \pm 6.87$ & 0 & $101.46 \pm 8.25$ & 1 \\
$\mathrm{Gr}-\mathrm{Ti}$ & $112.36 \pm 2.89$ & 0 & $118.43 \pm 7.22$ & 0 & $102.76 \pm 94.32$ & 0 \\
\hline
\end{tabular}

ODe is the average OD value of each experimental group. ODc is the average OD value of the control group. Cell toxicity grade (CTG) is obtained according to United States Pharmacopoeia standards [20]. After incubation for 24-72 h, all experimental groups were grade 0-1 (non-toxic).

\subsection{ALP Activity Detection}

As can be seen from Figure 6B, the ALP activity of the three groups increased gradually with the increase of culture time on $1 \mathrm{~d}, 4 \mathrm{~d}, 7 \mathrm{~d}$, and $10 \mathrm{~d}$. On day 1 of cell culture, ALP secretion was low, and there was no significant difference in ALP activity among all groups $(p>0.05)$. From the 4 th day on, ALP in each group increased gradually, and reached the highest value on the 10th day. The activity of ALP in the Ti group and Gr-Ti group was 
higher than that in the control group $(p<0.05)$, and the activity of ALP in the Gr-Ti group was higher than that in the Ti group.

\subsection{General Observation of Rabbit Mandible Model}

The experimental animals had normal movement and life except for the difficulty in feeding due to wound pain in the early postoperative period (the degree was mild and recovered a few days later). Both cheeks were slightly swollen after surgery, and the wound recovered well one week later without redness, swelling, exudation, or other inflammatory manifestations. The diet returned to normal three days after surgery. One week later, the wound healed without obvious signs of infection.

Figure 7 shows the samples after specimen removal.

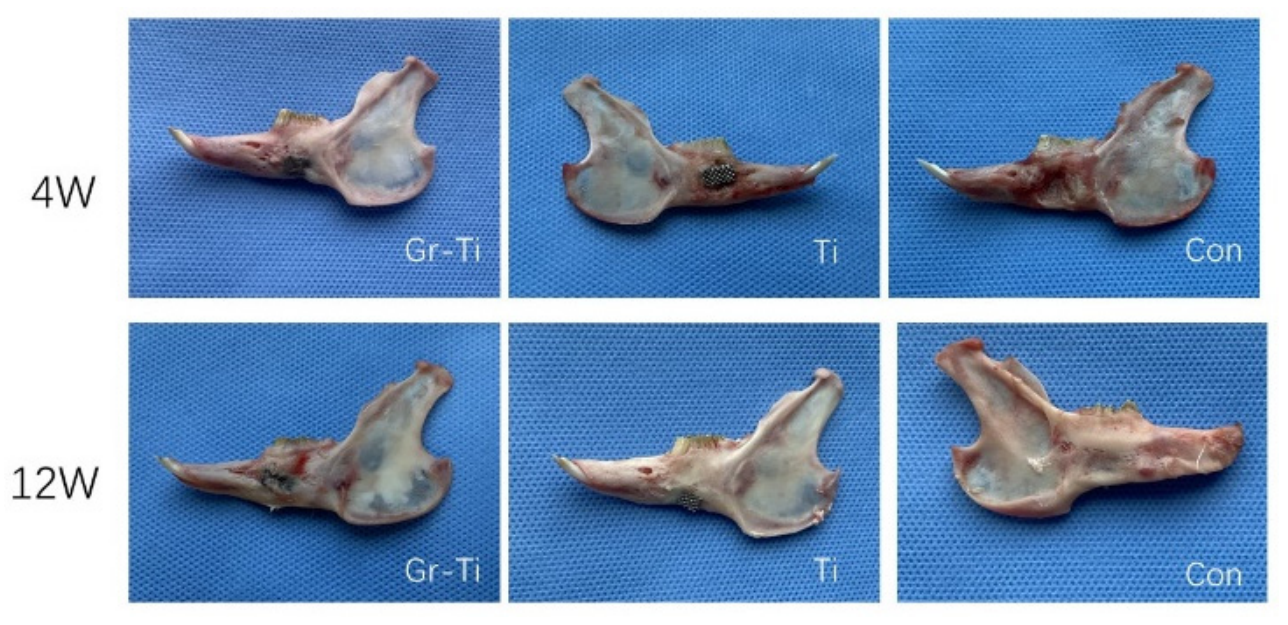

Figure 7. General observation of scaffold materials.

There was no prolapse of any scaffold. Four weeks after surgery, the implant material in groups A and B was soft and covered with fibrous connective tissue. The implant was loosely combined with the edge of the defect area. There was no significant difference between the two groups. In group $\mathrm{C}$, granulation tissue began to grow into the bone defect cavity. Twelve weeks after the operation, the implants in groups A and B were firmly connected to the edge of the defect area; the implants in group A were covered with new bone, and the new bone was hard and changed invisibly by pressing; the implants in group $B$ had no obvious boundary, and the surface part was covered by new bone, and the new bone was hard and changed invisibly by pressing; and the bone defect in group $\mathrm{C}$ was filled with granulation tissue and did not significantly reduce.

\subsection{Imaging Analysis (3D-CT)}

As can be seen from CT images (Figure 8), the bone defect area of the Gr-Ti scaffold was small 12 weeks after surgery, there was no obvious metal artifact around the bone tissue in the 3D images, and the bone tissue density was uniform, indicating that the new bone was closely connected with the surrounding area. In contrast, although new bone was generated in the Ti stent group, new bone only occurred at the defect edge, and the defect area was reduced, which was not enough to cover the whole defect area. Metal artifacts were seen locally, indicating that the osteogenic ability of the Ti stent group was weaker than that of the Gr-Ti stent group. In the blank control group, there was still no obvious new bone formation after 12 weeks, and no obvious signs of healing in the defect area. 

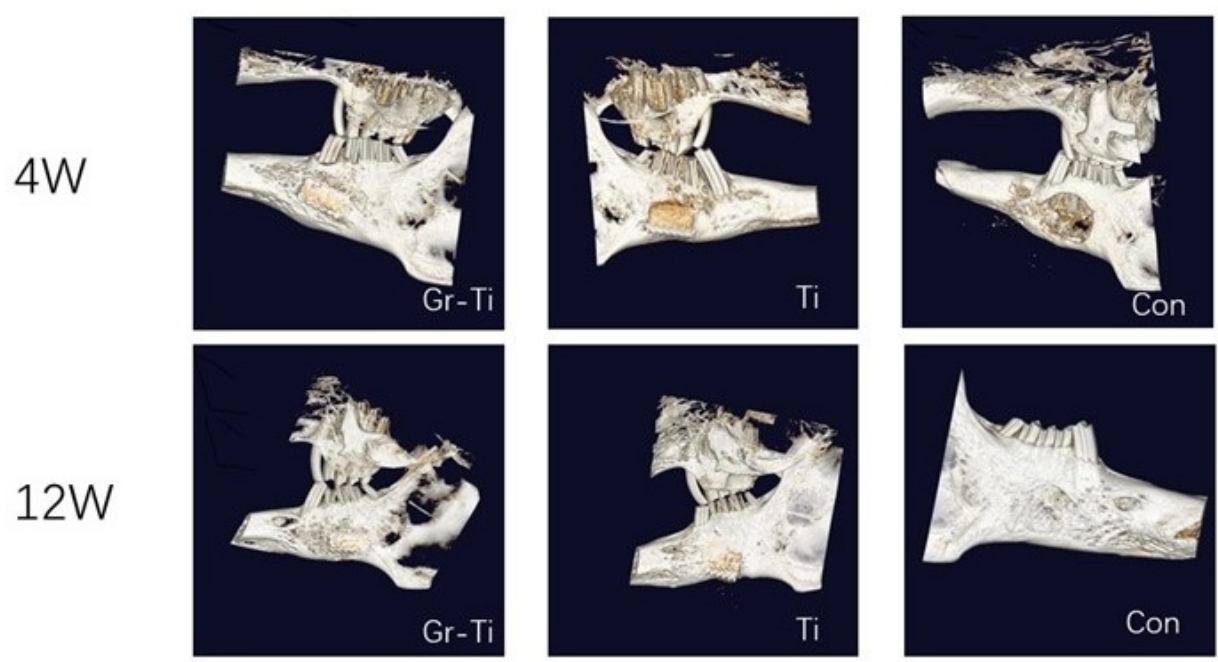

NBV

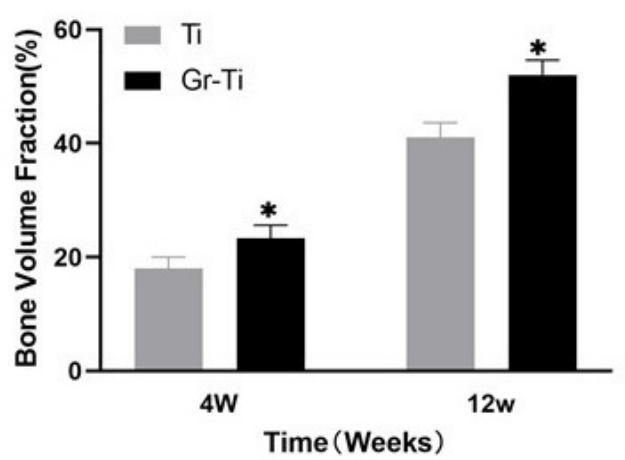

Figure 8. CT status and new bone density of scaffold materials. $\left({ }^{*} p<0.05\right)$.

The new bone was quantitatively determined by micro-CT software (Magics 13.0). Figure 8 shows the volume of new bone at each implant stage. It can be seen that 4 weeks after the Gr-Ti stent implantation, the amount of new bone increased significantly, which was much higher than that of the Ti stent group. At 12 weeks, NBV was the highest in the Gr-Ti group. These results confirm that the Gr-Ti scaffolds have higher osteogenic efficiency and good biocompatibility and osteogenic ability at the later stage of implantation. In group $\mathrm{B}$, no obvious bone formation was observed in NBV, and the total contribution to NBV was negligible.

\section{Discussion}

Studies have found that porous titanium alloy materials with a three-dimensional pore structure can effectively reduce the elastic modulus of the implant, make it match with normal bone tissue, and facilitate the growth of surrounding bone tissue into the implant to form mechanical locking, thus enhancing the fixation effect of the implant in the body [21] The mechanical and biological properties of porous titanium alloys are closely related to their pore structure and pore parameters. There have been many studies on the pore structure of scaffolds, and the pore size is still the most studied at present, because the long-term effect of scaffolds largely depends on the pore size. Studies have shown that with the increase of porosity and pore size, the elastic modulus and mechanical properties of porous materials decrease significantly [22]. However, if the porosity of the implant material is too high and the pore size is too large, the mechanical strength of the implant will decrease significantly and the support strength will be insufficient, resulting in the material being unusable for bone defect repair in the load-bearing part [23]. By analyzing and summarizing some relevant research results, we can find that the amount of 
bone tissue growth in porous materials is related to the pore size. In order to ensure the mechanical properties of a porous titanium implant and for new bone ingrowth material inside to form a strong mechanical locking, this topic put forward the optimization design of a porous titanium alloy stent, with a larger aperture (550 microns) and high porosity $(69 \%)$, up to the pore structure and pore parameters design standard. However, the optimal pore size for bone ingrowth is still controversial and the optimal pore size for promoting bone ingrowth remains to be further studied. The mechanical test results show that the compressive strength and elastic modulus of the porous titanium alloy implanted rod with this structure were $91 \pm 3 \mathrm{MPa}$ and $3.1 \pm 0.4 \mathrm{GPa}$, respectively, both of which were close to normal cortex bone, which could reduce the stress shielding effect and meet the structural support requirements of the load-bearing part [24], laying a good foundation for the next animal in vivo test. The elastic modulus of the Gr-Ti group and Ti group was measured, and there was no significant statistical difference between the two groups, indicating that the graphene coating prepared by us only improved the surface properties of Ti6Al4V, but had no significant impact on the overall mechanical properties of the substrate material. In conclusion, the porous titanium alloy material prepared in this study meets the requirements in pore design and mechanical properties, laying a good foundation for the next animal in vivo test. At the same time, SLM technology, an advanced rapid prototyping technology, is very suitable for the preparation of high-quality medical porous titanium alloy materials with a complex structure.

In 2004, Geim and Noveselov et al. from the university of Manchester in the United Kingdom isolated a two-dimensional graphene (graphene) material capable of stable existence for the first time $[25,26]$. Graphene's unique mechanical properties, such as high extensibility, flexibility, and adaptability to regular or irregular surfaces, are suitable for cell adhesion and migration and storage in stem cells for osteogenic differentiation $[27,28]$. The results of SEM and elemental analysis showed that the preparation of a graphene coating on a titanium alloy surface was successful. The coating and substrate material binding forces were very strong, stable, and not affected by ultrasonic shock and later cleaning, drying, and other processes. According to the later results of this experiment, this method is reliable and the coating can withstand high temperature and high pressure disinfection treatment, which is convenient for further biological study of the scaffold. Surface roughness is an important factor affecting the biological activity of biomaterials [29]. In this part of the experiment, we tested the surface roughness of graphene-coated titanium alloy and uncoated titanium alloy. The results show that the Ra of the Gr-Ti scaffold was $6.37 \pm 0.72 \mu \mathrm{m}$, and the surface roughness of the Ti scaffold was $4.95 \pm 1.12 \mu \mathrm{m}$, indicating that the graphene coating was successfully prepared on the surface of the porous titanium alloy by micro-arc oxidation technology. This not only gives the implant good biocompatibility, but also further promotes the effect of bone integration by increasing the surface roughness of the material.

As seed cells, the attachment ability of ADSCs on the material surface is one of the key factors for the success of material implantation. When evaluating the biological compatibility of the implanted material, an important indicator is to observe the ability of cells to adhere to the material surface and form good cell morphology [30]. In this experiment, we found that ADSCs grew well on the scaffold material, with a normal cell shape and surface morphology, and abundant connections between cells and between cells and the scaffold material (Figure 5), indicating that the Gr-Ti scaffold had good cellular compatibility and was suitable for the early adhesion growth of ADSCs. The adherent ADSCs showed pseudopod extension and intercellular connection, and firmly adhered to the scaffold. The intercellular connectivity and cell adhesion were considered as evidence for the survival and growth of ADSCs, and could also indirectly prove that graphene-coated porous titanium alloy scaffolds have no cytotoxic reactions [31].

ALP activity can reflect the mineralization ability of osteoblasts [32], and a CCK-8 test can reflect the proliferation ability of cells. It can be seen from the results of this study that, from the beginning of ADSCs entering the mature stage of the matrix, the ALP 
activity of both the Gr-Ti scaffold group and Ti scaffold group was higher than that of the blank control group, suggesting that the porous structure of the material may provide a three-dimensional space and a good growth environment for cell growth. Both scaffolds are safe and non-cytotoxic.

The critical bone defect animal model is mainly used to evaluate the repair effect of the repair materials used for the bone defect area [33]. In this experiment, a $0.8 \mathrm{~cm}$ full-thickness mandibular defect was prepared in the blank control group. After 12 weeks of observation, the bone defect in the control group was filled with fibrous tissue and adipose tissue and did not achieve clinical bone healing, so it could be defined as a critical bone defect [34]. Four weeks after surgery, there was a large area of low-density shadow around the Gr-Ti material, and the range of the low-density shadow was reduced in the proximal cortex, indicating a good effect of bone repair [35]. The bone volume fraction in the Gr-Ti group was significantly higher than that in the Ti group, indicating that the amount of new bone growth in the Gr-Ti material was significantly higher than that in the Ti group at 4 weeks after surgery. Twelve weeks after surgery, the low-density shadow area around Gr-Ti was further reduced, indicating that the bone defects around the material were repaired progressively with the increase of implantation time. The bone volume fraction in the Gr-Ti group increased to $49.42 \pm 3.28 \%$, which was much higher than that in the Ti group $(39.76 \pm 3.62 \%)$. This indicates that the amount of new bone ingrowth in the Gr-Ti group was significantly higher than that in group A. The results show that the graphene-coated porous titanium alloy scaffold had a good biological compatibility, and there was no obvious inflammatory reaction at the implant site. The new mineralized bone in the implant was deposited on the surface of the graphene-coated scaffold, which showed a good bone integration effect through the tight bonding between the coating and titanium alloy material.

To sum up, this study used advanced 3D metal printing technology for the preparation of the elastic modulus and bone tissue matching porous titanium alloy material; micro-arc oxidation technology was used in the construction of a bioactive graphene coating on the porous titanium alloy surface, and thus a metal material was created with high mechanical strength and an organic combination of bone conduction properties of the living bone. This provides a theoretical and experimental basis for the design of bone tissue engineering scaffolds and lays a solid foundation for promoting the clinical application of stem cells combined with bone tissue engineering.

\section{Conclusions}

In the present work, graphene was successfully fabricated on a 3D-printed titanium alloy, resulting in a better biocompatible surface. Graphene has favorable surface topography for improving the performance of titanium implants and shows immense potential for clinical application. The following main conclusions can be drawn:

1. SLM technology was used to design and prepare porous titanium alloy scaffolds with qualified porosity, pore size, and mechanical properties matching with bone tissue.

2. Compared with its performance on unmodified surfaces, coating surface composite graphene significantly increases the roughness of titanium alloy.

3. Graphene-coated porous titanium alloy scaffolds have a good biocompatibility and low toxicity for the growth, proliferation, and adhesion of human ADSCs.

4. The composite graphene-coated porous titanium alloy stent successfully repaired the rabbit mandibular defect, which laid a foundation for the clinical application of tissue engineering in the field of oral and maxillofacial bone defect repair.

Author Contributions: Conceptualization, X.S. and S.G.; methodology, S.T.; software, S.Y.; validation, S.G.; formal analysis, X.S. and S.T.; writing-original draft preparation, X.S.; writing-review and editing, S.T. All authors have read and agreed to the published version of the manuscript. 
Funding: This work was supported by the National Natural Science Foundation of China (51872332 and 81501857), the China Postdoctoral Science Foundation (2018M641741), and the Doctoral Research Initiation Fund of Liaoning Province (2019-BS-281). The funding had no role in the study design; in the collection, analyses, or interpretation of the data, or in the manuscript preparation.

Institutional Review Board Statement: The study was conducted according to the guidelines of the Declaration of Helsinki, and approved by the Ethics Committee of China Medical University (protocol code: CMU20211277; date of approval: 11 June 2021).

Informed Consent Statement: Not applicable.

Data Availability Statement: Not applicable.

Conflicts of Interest: The authors declare no conflict of interest.

\section{References}

1. Alraei, K.; Shrqawi, J.; Alarusi, K. Application of Recombinant Human BMP-2 with Bone Marrow Aspirate Concentrate and Platelet-Rich Fibrin in Titanium Mesh for Vertical Maxillary Defect Reconstruction prior to Implant Placement. Case Rep. Dent. 2021, 7, 2021. [CrossRef] [PubMed]

2. Adamička, M.; Adamičková, A.; Danišovič, L.; Gažová, A.; Kyselovič, J. Pharmacological Approaches and Regeneration of Bone Defects with Dental Pulp Stem Cells. Stem Cells Int. 2021, 2021, 4593322. [CrossRef] [PubMed]

3. Li, J.; Li, J.; Wei, Y.; Xu, N.; Li, J.; Pu, X.; Wang, J.; Huang, Z.; Liao, X.; Yin, G. Ion release behavior of vanadium-doped mesoporous bioactive glass particles and the effect of the released ions on osteogenic differentiation of BMSCs via the FAK/MAPK signaling pathway. J. Mater. Chem. B 2021, 9, 7848-7865. [CrossRef] [PubMed]

4. Prabha, R.D.; Kraft, D.C.E.; Harkness, L.; Melsen, B.; Varma, H.; Nair, P.D.; Kjems, J.; Kassem, M. Bioactive nano-fibrous scaffold for vascularized craniofacial bone regeneration. J. Tissue Eng. Regen. Med. 2018, 12, e1537-e1548. [CrossRef] [PubMed]

5. Mitra, I.; Bose, S.; Dernell, W.S.; Dasgupta, N.; Eckstrand, C.; Herrick, J.; Yaszemski, M.J.; Goodman, S.B.; Bandyopadhyay, A. 3D Printing in alloy design to improve biocompatibility in metallic implants. Mater. Today 2021, 45, 20-34. [CrossRef] [PubMed]

6. Oliver, J.N.; Su, Y.; Lu, X.; Kuo, P.; Du, J.; Zhu, D. Bioactive glass coatings on metallic implants for biomedical applications. Bioact. Mater. 2019, 4, 261-270. [CrossRef] [PubMed]

7. Gruenwald, W.; Bhattacharrya, M.; Jansen, D.; Reindl, L. Electromagnetic Analysis, Characterization and Discussion of Inductive Transmission Parameters for Titanium Based Housing Materials in Active Medical Implantable Devices. Materials 2018, 11, 2089. [CrossRef] [PubMed]

8. Su, Y.; Luo, C.; Zhang, Z.; Hermawan, H.; Zhu, D.; Huang, J.; Liang, Y.; Li, G.; Ren, L. Bioinspired surface functionalization of metallic biomaterials. J. Mech. Behav. Biomed. Mater. 2018, 77, 90-105. [CrossRef] [PubMed]

9. Zhao, H.; Shen, S.; Zhao, L.; Xu, Y.; Li, Y.; Zhuo, N. 3D printing of dual-cell delivery titanium alloy scaffolds for improving osseointegration through enhancing angiogenesis and osteogenesis. BMC Musculoskelet. Disord. 2021, 22, 734. [CrossRef] [PubMed]

10. Mehboob, H.; Tarlochan, F.; Mehboob, A.; Chang, S.; Ramesh, S.; Harun, W.S.W.; Kadirgama, K. A novel design, analysis and 3D printing of Ti-6Al-4V alloy bio-inspired porous femoral stem. J. Mater. Sci. Mater. Med. 2020, 31, 78. [CrossRef] [PubMed]

11. Wang, H.; Su, K.; Su, L.; Liang, P.; Ji, P.; Wang, C. Comparison of 3D-printed porous tantalum and titanium scaffolds on osteointegration and osteogenesis. Mater. Sci. Eng. C Mater. Biol. Appl. 2019, 104, 109908. [CrossRef]

12. Pereira, A.T.; Schneider, K.H.; Henriques, P.C.; Grasl, C.; Melo, S.F.; Fernandes, I.P.; Kiss, H.; Martins, M.C.L.; Bergmeister, H.; Gonçalves, I.C. Graphene Oxide Coating Improves the Mechanical and Biological Properties of Decellularized Umbilical Cord Arteries. ACS Appl. Mater. Interfaces 2021, 13, 32662-32672. [CrossRef]

13. Lu, J.; Sun, J.; Zou, D.; Song, J.; Yang, S. Graphene-Modified Titanium Surface Enhances Local Growth Factor Adsorption and Promotes Osteogenic Differentiation of Bone Marrow Stromal Cells. Front. Bioeng. Biotechnol. 2020, 8, 621788. [CrossRef] [PubMed]

14. Azadian, E.; Arjmand, B.; Ardeshirylajimi, A.; Hosseinzadeh, S.; Omidi, M.; Khojasteh, A. Polyvinyl alcohol modified polyvinylidene fluoride-graphene oxide scaffold promotes osteogenic differentiation potential of human induced pluripotent stem cells. $J$. Cell. Biochem. 2020, 121, 3185-3196. [CrossRef] [PubMed]

15. Wu, Y.; Wang, Y.; Tian, S.; Li, H.; Zhao, Y.; Jia, D.; Zhou, Y. Formation mechanism, degradation behavior, and cytocompatibility of a double-layered structural MAO/rGO-CaP coating on AZ31 Mg. Colloids Surf. B Biointerfaces 2020, 190, 110901. [CrossRef] [PubMed]

16. Zhang, J.; Liu, Y.; Chen, Y.; Yuan, L.; Liu, H.; Wang, J.; Liu, Q.; Zhang, Y. Adipose-Derived Stem Cells: Current Applications and Future Directions in the Regeneration of Multiple Tissues. Stem Cells Int. 2020, 2020, 8810813. [CrossRef] [PubMed]

17. Wang, Z.; Han, L.; Sun, T.; Wang, W.; Li, X.; Wu, B. Osteogenic and angiogenic lineage differentiated adipose-derived stem cells for bone regeneration of calvarial defects in rabbits. J. Biomed. Mater. Res. A 2021, 109, 538-550. [CrossRef]

18. Singh, A.V.; Rosenkranz, D.; Ansari, M.H.D.; Singh, R.; Kanase, A.; Singh, S.P.; Johnston, B.; Tentschert, J.; Laux, P.; Luch, A. Artificial Intelligence and Machine Learning Empower Advanced Biomedical Material Design to Toxicity Prediction. Adv. Intell. Syst. 2020, 2, 2000084. [CrossRef] 
19. Arjunan, A.; Demetriou, M.; Baroutaji, A.; Wang, C. Mechanical performance of highly permeable laser melted Ti6Al4V bone scaffolds. J. Mech. Behav. Biomed. Mater. 2020, 102, 103517. [CrossRef]

20. Tamashiro, N.S.M.; Souza, R.Q.; Gonçalves, C.R.; Ikeda, T.I.; Luz, R.A.; Cruz, A.S.; Padoveze, M.C.; Graziano, K.U. Cytotoxicity of cannulas for ophthalmic surgery after cleaning and sterilization: Evaluation of the use of enzymatic detergent to remove residual ophthalmic viscosurgical device material. J. Cataract. Refract. Surg. 2013, 39, 937-941. [CrossRef]

21. Xiong, Y.Z.; Gao, R.N.; Zhang, H.; Dong, L.L.; Li, J.T.; Li, X. Rationally designed functionally graded porous Ti6Al4V scaffolds with high strength and toughness built via selective laser melting for load-bearing orthopedic applications. J. Mech. Behav. Biomed. Mater. 2020, 104, 103673. [CrossRef]

22. Ran, Q.; Yang, W.; Hu, Y.; Shen, X.; Yu, Y.; Xiang, Y.; Cai, K. Osteogenesis of 3D printed porous Ti6Al4V implants with different pore sizes. J. Mech. Behav. Biomed. Mater. 2018, 84,1-11. [CrossRef]

23. Luo, C.; Wang, C.; Wu, X.; Xie, X.; Wang, C.; Zhao, C.; Zou, C.; Lv, F.; Huang, W.; Liao, J. Influence of porous tantalum scaffold pore size on osteogenesis and osteointegration: A comprehensive study based on 3D-printing technology. Mater. Sci. Eng. C Mater. Biol. Appl. 2021, 129, 112382. [CrossRef] [PubMed]

24. Wang, H.; Su, K.; Su, L.; Liang, P.; Ji, P.; Wang, C. The effect of 3D-printed Ti6Al4V scaffolds with various macropore structures on osteointegration and osteogenesis: A biomechanical evaluation. J. Mech. Behav. Biomed. Mater. 2018, 88, 488-496. [CrossRef] [PubMed]

25. Novoselov, K.S.; Geim, A.K.; Morozov, S.V.; Jiang, D.; Zhang, Y.; Dubonos, S.V.; Grigorieva, I.V.; Firsov, A.A. Electric field effect in atomically thin carbon films. Science 2004, 306, 569. [CrossRef]

26. Lee, C.; Wei, X.; Kysar, J.W.; Hone, J. Measurement of the elastic properties and intrinsic strength of monolayer graphene. Science 2008, 321, 385-388. [CrossRef]

27. Xie, H.; Cao, T.; Franco-Obregón, A.; Rosa, V. Graphene-Induced Osteogenic Differentiation Is Mediated by the Integrin/FAK Axis. Int. J. Mol. Sci. 2019, 20, 574. [CrossRef] [PubMed]

28. Zhu, J.Q.; Li, B.; Xu, M.; Liu, R.; Xia, T.; Zhang, Z.H.; Xu, Y.; Liu, S.J. Graphene Oxide Promotes Cancer Metastasis through Associating with Plasma Membrane to Promote TGF- $\beta$ Signaling-Dependent Epithelial-Mesenchymal Transition. ACS Nano 2020, 14, 818-827. [CrossRef]

29. Zou, J.; Wang, W.W.; Nie, Y.; Xu, X.; Ma, N.; Lendlein, A. Microscale roughness regulates laminin-5 secretion of bone marrow mesenchymal stem cells. Clin. Hemorheol. Microcirc. 2019, 73, 237-247. [CrossRef] [PubMed]

30. Liu, J.; Zhou, P.; Long, Y.; Huang, C.X.; Chen, D.N. Repair of bone defects in rat radii with a composite of allogeneic adiposederived stem cells and heterogeneous deproteinized bone. Stem Cell Res. Ther. 2018, 9, 79. [CrossRef]

31. Ajay, V.S.; Romi, S.M.; Harald, J.; Heike, R.; Yves, U.H.; Philipp, R.; Frank, B.; Katherina, S.; Ashish, G.; Peter, L.; et al. Evaluating Particle Emissions and Toxicity of 3D Pen Printed Filaments with Metal Nanoparticles as Additives: In Vitro and in Silico Discriminant Function Analysis. ACS Sustain. Chem. Eng. 2021, 9, 11724-11737. [CrossRef]

32. Wang, Q.; Miao, Y.M.; Qian, Z.Y.; Chen, L.D.; Lu, T.; Xu, Y.; Jiang, X.W.; Shen, Y.C. MicroRNA-15a-5p plays a role in osteogenic MC3T3-E1 cells differentiation by targeting PDCD4 (programmed cell death 4 ) via Wnt/ $\beta$-catenin dependent signaling pathway. Bioengineered 2021, 12, 8173-8185. [CrossRef] [PubMed]

33. de Souza, L.P.L.; Lopes, J.H.; Ferreira, F.V.; Martin, R.A.; Bertran, C.A.; Camilli, J.A. Evaluation of effectiveness of $45 \mathrm{~S} 5$ bioglass doped with niobium for repairing critical-sized bone defect in in vitro and in vivo models. J. Biomed. Mater. Res. A 2020, 108, 446-457. [CrossRef] [PubMed]

34. Cheng, Z.A.; Alba-Perez, A.; Gonzalez-Garcia, C.; Donnelly, H.; Llopis-Hernandez, V.; Jayawarna, V.; Childs, P.; Shields, D.W.; Cantini, M.; Ruiz-Cantu, L.; et al. Nanoscale Coatings for Ultralow Dose BMP-2-Driven Regeneration of Critical-Sized Bone Defects. Adv. Sci. 2019, 6, 1800361. [CrossRef] [PubMed]

35. Neuber, C.; Schulze, S.; Förster, Y.; Hofheinz, F.; Wodke, J.; Möller, S.; Schnabelrauch, M.; Hintze, V.; Scharnweber, D.; Rammelt, S.; et al. Biomaterials in repairing rat femoral defects: In vivo insights from small animal positron emission tomography/computed tomography (PET/CT) studies. Clin. Hemorheol. Microcirc. 2019, 73, 177-194. [CrossRef] 